\title{
Equivariant Constrained Symplectic Integration
}

\author{
Robert I. McLachlan ${ }^{1}$ and Clint Scovel ${ }^{2}$ \\ 1 Program in Applied Mathematics \\ University of Colorado at Boulder \\ Boulder CO 80309-0526 (rxm@boulder.colorado.edu) \\ 2 Computer Research Group, C-3, MS-B265 \\ Los Alamos National Laboratory, \\ Los Alamos, NM 87545 (jcs@cabron.c3.lanl.gov)
}

10 March 1994

\begin{abstract}
We use recent results on symplectic integration of Hamiltonian systems with constraints to construct symplectic integrators on cotangent bundles of manifolds by embedding the manifold in a linear space. We also prove that these methods are equivariant under cotangent lifts of a symmetry group acting linearly on the ambient space and consequently preserve the corresponding momentum. These results provide an elementary construction of symplectic integrators for LiePoisson systems and other Hamiltonian systems with symmetry. The methods are illustrated on the free rigid body, the heavy top, and the double spherical pendulum.
\end{abstract}

\section{Introduction}

Much work has been done on symplectic integrators on linear phase spaces such as $\mathbb{R}^{2 n}$ with the standard symplectic structure; see Sanz-Serna [25], Scovel [26], and Yoshida [32] for reviews. Symplectic methods on curved symplectic manifolds, such as cotangent bundles of Lie groups and coadjoint orbits, tend to be complicated due to the fact that one must coordinatize the manifold and these coordinate charts can make the integrator inefficient. It is a standard practice in numerical analysis to embed the manifold in a Euclidean space to take advantage of the simplicity of function evaluations and then constrain the motion to remain on the manifold. In this case we also require that the mapping induced on the underlying symplectic manifold be symplectic.

This is the same idea used in Lagrangian mechanics with holonomic constraints. One can either go to coordinates on the constraint submanifold, where the motion is determined by the Euler-Lagrange equations, or one can write down the Euler-Lagrange equations on the full space and add Lagrange multipliers so that the dynamics maintains the constraints. The two approaches are equivalent in that they give the same equations of motion, but are not necessarily equivalent in complexity.

However, this still leaves open the relationship between the motion on the constraint surface and that on the cotangent bundle of the constrained configu- 
ration variables. We prove a theorem establishing this connection. An alternative treatment can be found in Marsden and Ratiu [16, §8.3].

Having prepared the geometrical setting, we investigate in depth a constraint algorithm due to Reich [21]. This preserves the standard symplectic form restricted to the constraint surface. There are other algorithms that also do this: for simple mechanical systems (when the Hamiltonian is the sum of a quadratic kinetic energy and a potential), the "RATTLE" method of molecular dynamics [2] was shown to be symplectic in this sense by Leimkuhler and Skeel [11]; Jay [10] gives a class of suitable partitioned Runge-Kutta methods, one of which reduces to RATTLE in the case of a simple mechanical system. The problem for more general constraints is still open.

In holonomically constrained systems, the primary constraints on the configuration variables entail (via the theory of Dirac constraints) the secondary constraint that the time derivative of the primary constraints also vanishes. All of the above algorithms satisfy the secondary constraint in a step which, as is shown in [11] for the case of simple mechanical systems, is a projection onto the constraints. We show in section 5 how the introduction of a metric (and reformulation of the algorithm on the tangent, instead of cotangent, bundle of the ambient space) generalizes this notion. The choice of an appropriate metric can also simplify the algorithm, and brings out connections with traditional presentations of classic dynamical systems such as the free rigid body.

For Hamiltonian systems with symmetry, it is desirable to construct a symplectic integrator that preserves the corresponding momentum [1]. Consider for example the free rigid body with configuration space $S O(n)$. Lewis and Simo [12] construct momentum- and energy-preserving integrators for this system; only for $n=3$ is one of them also symplectic. Moser and Veselov [19] provide a completely integrable momentum-preserving symplectic integrator for arbitrary $n$ by discretizing the Lagrangian formulation, but it is not clear that the method is useful for general Hamiltonian systems. (Their interest was in integrable mappings rather than in numerical methods per se.)

If one is not interested in the orbital position corresponding to the symmetry, it may be possible to construct a symplectic integrator on the Poisson or symplectic reduced space. See Marsden and Ratiu [15] for a general treatment of Poisson reduction. The Poisson reduction of the cotangent bundle of a Lie group with respect to the group's natural action on itself is the dual of the Lie algebra with the Lie-Poisson structure. This space is foliated by the coadjoint orbits which are the symplectic leaves of the Poisson manifold. It is apparent that symplectic integration on these leaves might be a difficult matter. However, Ge and Marsden [6] do construct Lie-Poisson integrators, those which preserve the leaves and are symplectic on them, and Ge [8] generalizes this result to Poisson manifolds. Channell and Scovel [3] simplify the Ge-Marsden technique by transforming the generating equations to the dual of the Lie algebra. They developed and tested Lie-Poisson integrators of arbitrary order for Lie algebras with positive-definite $A d$-invariant quadratic form and first order Lie-Poisson integrators for arbitrary Lie algebras. These could be extended to higher order by composition $[28,31]$, but unfortunately they are not very efficient.

Consequently, is it is desirable to construct a general constraint algorithm which is symplectic and equivariant (respectively momentum preserving) and so 
projects to a Poisson (respectively symplectic) integrator on the reduced spaces. In this direction, we show in section 4 that the constraint algorithm considered here solves this problem for cotangent lift symmetries which act linearly on the ambient space (see Reich [23] for an alternative treatment). This result provides an elementary construction of momentum-preserving symplectic integrators and, in particular, an elementary construction of Lie-Poisson integrators.

For simple mechanical systems, Leimkuhler and Skeel [11] show that it is not really necessary to enforce the secondary constraints, except possibly when output is required. The update of the configuration variables is unchanged even if this step is dropped. But even in this special case, in the presence of symmetry, the projection onto the secondary constraints is crucial; it allows Lie-Poisson or semi-direct reduction to apply to the algorithm.

One advantage of the present approach to Lie-Poisson integration is that it does not require the Lie algebra to be written as a matrix Lie algebra. However, it does require a description of the Lie group as embedded in a linear space. We illustrate this construction for an algebra arising when a density distribution is approximated by a finite set of moments. It also requires various nondegeneracy and invariance conditions on the constraints and on the extension of the Hamiltonian to the ambient space, which we point out during the presentation.

To illustrate the different spaces and variants of the algorithms, in section 6 we construct them in detail for the free rigid body, the heavy top, and the double spherical pendulum and confirm the theoretical results. We are not proposing the constraint approach as the ideal numerical method here; for practical purposes one should also consider that many Lie-Poisson systems, including rigid bodies, can be explicitly symplecticly integrated directly in the reduced phase space, by splitting the Hamiltonian [17, 22]. But curiously, it turns out that the constraint integrator we obtain for the free rigid body is that of Moser and Veselov [19] - a completely integrable mapping preserving all the integrals of the continuous system. The reason why discretizing the Lagrangian description and then mapping to the Hamiltonian setting produces the same algorithm as mapping to the Hamiltonian setting and then applying the constraint algorithm remains unclear.

\section{Holonomic constraints}

Consider a linear configuration space $M$ and configuration constraint manifold $N \subset M$ defined by $\phi=0$, where $\phi: M \rightarrow V$ and $V$ is a linear space. We are free to assume an inner product on $V$ and denote by $d \phi(q): T_{q} M \rightarrow V$ the linearization of $\phi$ and $d \phi(q)^{*}: V \rightarrow T_{q}^{*} M$ its adjoint defined by

$$
d \phi(q)^{*} \mu(\dot{q})=\langle\mu, d \phi(q) \dot{q}\rangle,
$$

where we have used the usual identification of fibers of $T V$ with $V$. Suppose that we have a nondegenerate Lagrangian on $T M$. The Euler-Lagrange equations for the motion on $T M$ do not preserve $\phi=0$. To constrain the configuration by the holonomic constraints $\phi=0$, one can proceed in two fashions. Firstly, one can use the method of Lagrange multipliers for the Euler-Lagrange equations on $T M$ so that the motion stays in $T N$, where the multipliers provide the constraining 
force. Secondly, one can simply use coordinates in $T N$ and compute the EulerLagrange equations on $T N$. The two methods are equivalent (Symon [29]). To obtain the Hamiltonian picture, we use the Legendre transforms to map to $T^{*} M$ and $T^{*} N$. In the Dirac theory of constraints, a constraint surface is described in terms of constraint functions. The Hamiltonian is the restricted Hamiltonian and the constraint symplectic form is the standard symplectic two-form restricted to the constraint surface, if it is nondegenerate. It is automatically closed, and is nondegenerate if the constraint surface is cosymplectic: namely, if the matrix

$$
\left\{\lambda_{i}, \lambda_{j}\right\}
$$

is invertible where the $\lambda_{i}$ are the constraints. The cosymplectic condition ensures that the constrained dynamics are well posed and that the constraint functions are nondegenerate.

We shall see in Theorem 1 that the constraints, and hence the cosymplectic condition, depend on the Hamiltonian, confirming the notion that in the Lagrangian setting the constraint forces and the motion must be determined simultaneously. Although the symplectic structure is obtained simply by restriction to the constraint surface, the Poisson bracket on the constraint manifold has to be modified as in equation (1) below if it is be evaluated on functions defined on the ambient space $T^{*} M$. One can think of the new terms as canceling the contribution to the bracket from the derivatives of the arguments in directions not tangent to the constraint surface. See [9, p.236] for more details.

Given the Dirac theory of constraints, what are the appropriate constraints on $T^{*} M$ and the appropriate map from the constraint manifold to $T^{*} N$ so that the constrained motion maps to that on $T^{*} N$ ? Reich [21] calculates the Hamiltonian equations of constrained motion on the constraint surface $\phi_{1}=\phi=0$ and $\phi_{2}=\{H, \phi\}=0$. However, the relationship between this flow and the natural one on $T^{*} N$ is left unclear. We call $H$ cosymplectic with respect to the primary constraints $\phi$ if the constraint manifold $\phi_{1}=\phi_{2}=0$ is cosymplectic.

A crucial ingredient of the following theorem, and indeed of our whole presentation, is the following map $\psi$ induced from the embedding $N \rightarrow M$ :

$$
\begin{aligned}
\psi:\left.T^{*} M\right|_{N} & \rightarrow T^{*} N \\
(q, p) & \mapsto\left(q, p_{-}\right)
\end{aligned}
$$

where $\left.T^{*} M\right|_{N}$ is the bundle over $N$ with fibers the cotangent fibers of $T^{*} M$, and $p_{-}$is the covector $p$ restricted to $T_{q} N$. It is linear in $p$, and the range of $d \phi(q)^{*}$ is in the kernel of $\psi$. For, let $p=d \phi(q)^{*} \mu$. Then $p_{-}(\dot{q})=\left(\left.d \phi(q)^{*} \mu\right|_{T_{q} N}\right)(\dot{q})$ $=\langle\mu, d \phi(q) \dot{q}\rangle=0$ because $\dot{q} \in T_{q} N$. In fact, the cosymplectic condition implies the nondegeneracy of $\phi$, i.e., that range $\left(d \phi(q)^{*}\right)=\operatorname{ker}(\psi)$. Because $\psi$ does not alter its first argument, we shall sometimes write $p_{-}=\psi(q, p)$.

Theorem 1. Suppose that the Hamiltonian system on $T^{*} N$ arises from a nondegenerate Lagrangian $L$ on $T N$ which can be extended to a nondegenerate Lagrangian L on TM. Suppose the Legendre transform determines a Hamiltonian system on $T^{*} M$ which is cosymplectic with respect to $\phi$. The dynamics on the constraint surface

$$
C M=\left\{(q, p): \phi_{1}=\phi=0, \phi_{2}=\{H, \phi\}=0\right\}
$$

is conjugate by the map $\psi$ to that on $T^{*} N$. 
Proof. The constrained Euler-Lagrange equations are

$$
\frac{d}{d t}\left(\partial_{\dot{q}} L\right)-\partial_{q} L=d \phi^{*} \mu
$$

where the multipliers $\mu$ are determined by differentiation of the secondary constraints $d \phi \dot{q}=0$. Now consider the Dirac bracket evaluated on $H$ :

$$
\{H, F\}_{c}=\{H, F\}+\left\{H, \lambda_{i}\right\} C^{i j}\left\{\lambda_{j}, F\right\}
$$

where $\lambda_{i}=\left(\phi_{1}\right)_{i}, \lambda_{i+n}=\left(\phi_{2}\right)_{i}$ for $i=1, \ldots, n=\operatorname{dim} V$. Subscripts $i_{1}$ will be in the range 1 to $n$ and refer to the primary constraints; subscripts $i_{2}$ will be in the range $n+1$ to $2 n$ and refer to secondary constraints. $C$ is the inverse of the matrix $\left\{\lambda_{i}, \lambda_{j}\right\}$. Since $\phi_{2}=\{H, \phi\}$, on the constraint manifold this expression becomes

$$
\{H, F\}_{c}=\{H, F\}+\left\{H, \phi_{2 i_{2}}\right\} C^{i_{2} j}\left\{\lambda_{j}, F\right\} .
$$

The matrix $\left\{\lambda_{i}, \lambda_{j}\right\}$ has the form

$$
\left(\begin{array}{cc}
0 & a \\
-a^{t} & b
\end{array}\right)
$$

and so has the inverse

$$
\left(\begin{array}{cc}
a^{-t} b a^{-1} & -a^{-t} \\
a^{-t} & 0
\end{array}\right)
$$

if $a=d \phi\left(\partial^{2} H / \partial p^{2}\right) d \phi^{*}$ is invertible. This is Reich's [21] nondegeneracy condition and is equivalent to the cosymplectic condition that $\left\{\lambda_{i}, \lambda_{j}\right\}$ be invertible. Consequently, $C^{i_{2} j_{2}}=0$, so that the bracket becomes

$$
\{H, F\}_{c}=\{H, F\}+\left\{H, \phi_{2 i_{2}}\right\} C^{i_{2} j_{1}}\left\{\phi_{j_{1}}, F\right\} .
$$

Therefore the constrained equations of motion are

$$
\begin{aligned}
& \dot{q}=\partial_{p} H \\
& \dot{p}=-\partial_{q} H+d \phi^{*} \mu .
\end{aligned}
$$

Since $\mu$ is determined by setting $d\{H, \phi\} / d t=0$ which is the same as $d(d \phi \dot{q}) / d t=$ 0 , and $\partial_{q} H=-\partial_{q} L$ and $p=\partial_{\dot{q}} L$ we find that the constrained Euler-Lagrange equations and constrained Hamiltonian equations give the same motion. Since the Euler-Lagrange equations on $T M$ with constraints to $T N$ are equivalent to the Euler-Lagrange equations on $T N$, and the latter are equivalent to the Hamiltonian system on $T^{*} N$, we are done with the proof that these are the correct constraints.

What is left is to show that $\psi$ is the isomorphism of the constraint surface with $T^{*} N$. The constraint manifold is the image of the Legendre transform evaluated on $T N$. Namely, $C M=F L(T N)$, since the constraints are equivalent to restriction to $T N$ in the Lagrangian picture and the Lagrangian is nondegenerate on $T M$ ([1, p. 208]). Denote by $\left.\psi\right|_{C M}$ the map $\psi$ with domain restricted to $C M$. The Legendre transform on $T M$ is $p=\partial_{\dot{q}} L$ and on $T N$ is $p_{-}=\psi\left(q,\left.\partial_{\dot{q}} L\right|_{T N}\right)$. Let $h$ denote the Hamiltonian restricted to $C M$ and $H$ the Hamiltonian on $T^{*} N$. 
We show that $\left.\psi\right|_{C M}$ is symplectic and $h=\left.H \circ \psi\right|_{C M}$, which will establish the conjugacy of the respective dynamics under $\psi[1$, p.194].

Let $p d q_{C M}$ denote the canonical one form on $T^{*} M$ restricted to $C M$. The values of the one form are $\left.p d q\right|_{C M}(\dot{q}, \dot{p})=p(\dot{q})$. The canonical one form on $T^{*} N$ is $p_{-} d q$ and has values $p_{-} d q(\dot{q}, \dot{p})=p_{-}(\dot{q})$. In both cases $\dot{q}$ is tangent to $N$, so that $\left.p d q\right|_{C M}(\dot{q}, \dot{p})=p(\dot{q})=p_{-}(\dot{q})$ and since the cosymplectic condition implies that $\left.\psi\right|_{C M}$ is a diffeomorphism, $\psi^{*}\left(p_{-} d q\right)=\left.p d q\right|_{C M}$. Since pullbacks and restrictions commute with exterior differentiation, $\left.\psi\right|_{C M}$ is symplectic.

On $C M$, the Hamiltonian is

$$
H(q, p)=p(\dot{q})-L(q, \dot{q})
$$

where $\dot{q}$ is determined as a function of $q$ and $p$; on $T^{*} N$, it is

$$
H\left(q, p_{-}\right)=p_{-}(\dot{q})-L(q, \dot{q})
$$

Since $p(\dot{q})=p_{-}(\dot{q})$ for $\dot{q} \in T N$, and the respective $\dot{q}$ 's will be the same if $\left(q, p_{-}\right)=\left.\psi\right|_{C M}(q, p), h=\left.H \circ \psi\right|_{C M}$. The proof is finished.

\section{The constraint algorithm}

Reich [21] considers the following algorithm and shows it to be symplectic. Begin with $(q, p) \in C M ; t$ is the time step. Solve

$$
\begin{aligned}
Q & =q+t \partial_{p} H(q, \tilde{p}) \\
\tilde{p} & =p-t\left(\partial_{q} H(q, \tilde{p})-d \phi(q)^{*} \mu\right) \\
\phi(Q) & =0
\end{aligned}
$$

where $\mu$ is determined by the condition that $\phi(Q)=0$. Then, solve

$$
\begin{aligned}
P & =\tilde{p}+t d \phi(Q)^{*} \nu \\
\phi_{2}(Q, P) & =0
\end{aligned}
$$

where $\nu$ is determined by the condition that $\phi_{2}(Q, P)=0$.

Note that this determines a map from $C M$ to itself, but does not require the intermediate variable $\tilde{p}$ to be so constrained. This algorithm is not only symplectic but momentum preserving for cotangent lifts of linear symmetry actions, as Theorem 2 below shows. However, first we show how the algorithm appears on $T^{*} N$.

Again the intermediate variable $\tilde{p}$ lies in $\left.T^{*} M\right|_{N}$. Recall that the range of $d \phi^{*}$ is the kernel of $\psi$, so that applying $\psi$ to the constraint algorithm $(2,3)$ eliminates the Lagrange multipliers $\mu$ and $\nu$ and produces the following algorithm.

Begin with $(q, p) \in T^{*} N$. Solve

$$
\begin{aligned}
Q & =q+t \partial_{p} H(q, \tilde{p}) \\
\psi(q, \tilde{p}) & =p-t \psi\left(q, \partial_{q} H(q, \tilde{p})\right) \\
\phi(Q) & =0
\end{aligned}
$$

where $\tilde{p}$ is determined so that $\phi(Q)=0$; note that $\psi\left(q, \partial_{q} H\right)=\left.\partial_{q} H\right|_{T N}$. Then, compute

$$
P=\psi(Q, \tilde{p}) .
$$


Theorem 2. Suppose that the constraint function $\phi$ is invariant under a symmetry group $G$ acting linearly on $M$, and that the Hamiltonian $H$ is invariant under cotangent lifts of $G$ acting on $T^{*} M$ and is cosymplectic with respect to $\phi$. Then, the constraint algorithm is first order, symplectic, G-equivariant, and, if the algorithm has a fixed point, preserves the corresponding momentum.

Proof. The constraint algorithm $(2,3)$ can be written in generating function form as

$$
\begin{aligned}
Q d \tilde{p}+p d q+t d \phi(q)^{*} \mu & =d S_{1}(q, \tilde{p}) \\
\phi(Q) & =0
\end{aligned}
$$

where $S_{1}(q, \tilde{p})=\tilde{p}(q)+H(q, \tilde{p})$, followed by

$$
\begin{aligned}
\tilde{p} d Q-P d Q+t d \phi(Q)^{*} \nu & =d S_{2}(Q, \tilde{p}) \\
\phi_{2}(Q, P) & =0
\end{aligned}
$$

where $S_{2}(Q, \tilde{p})=0$.

The generating equations $(6,7)$ without the constraints, and $\mu$ and $\nu$ fixed parameters, determine the map $\left((2,3)\right.$ without constraints) from $T^{*} M$ to itself. Since the forms $Q d \tilde{p}, p d q, P d Q, \tilde{p} d Q, \tilde{p}(q), d \phi(q)^{*} \mu$, and $d \phi(Q)^{*} \nu$ are invariant under cotangent lifts of linear actions and $H$ is assumed invariant under the symmetry group, this map is equivariant. Since the constraints are invariant under the symmetry group and the Hamiltonian satisfies the cosymplectic condition, it follows that the map, determined by choosing the parameters $\mu$ and $\nu$ so that the constraints are satisfied by $(Q, P)$ when the initial conditions satisfy them, is also equivariant. It also follows that the determined functions $\mu(q, p)$ and $\nu(q, p)$ are invariant.

Since the pullback to the constraint manifold commutes with the exterior derivative, the first equations state that the map $(q, p) \mapsto(Q, \tilde{p})$ is symplectic from the constraint manifold to its image with respect to the restricted forms. The second equations state that the map $(Q, \tilde{p}) \mapsto(Q, P)$ from this image to the constraint manifold is symplectic. Since the restricted form is the symplectic form for constrained dynamics, the composition of (6) and (7) is symplectic.

Finally, by the equivalence theorem of Feng and Ge [5], if the algorithm has a fixed point then the momentum is preserved.

The generating function representation provides a slightly different proof of the symplecticity of this algorithm than found in Reich [21] and suggests higherorder methods based on generating function theory.

In many situations, we begin with a Hamiltonian system on $T^{*} N$ but do not wish to attempt to coordinatize $N$. We may wish to integrate in a linear space with constraints. We therefore state some consequences of the above theorems.

Corollary 3. Suppose that the Hamiltonian on $T^{*} N$ has a group of tangent lift symmetries $G$ which can be extended to act linearly on $M$. Suppose the Hamiltonian has a nondegenerate Legendre transform and that the corresponding Lagrangian on $T N$ has a nondegenerate, $G$-invariant extension to $T M$ such that the corresponding Hamiltonian on $T^{*} M$ is cosymplectic with respect to $\phi$. Then, the constraint algorithm projects to a first order, $G$-equivariant, symplectic integrator on $T^{*} N$. If the algorithm has a fixed point then it preserves the momentum. 
Proof. The proof follows directly from Theorem 2 since the map $\left.\psi\right|_{C M}$ is equivariant, symplectic, and maps to the correct Hamiltonian.

The condition that the algorithm have a fixed point is satisfied by many Hamiltonians including those examples treated in section 6. In particular, these algorithms, applied to any Hamiltonian which has a quadratic kinetic energy function, have fixed points and so the method preserves the momentum. However, it should be noted that even if the momentum were not preserved, the integrator would be a Poisson map on the Poisson-reduced space but might not preserve the symplectic leaves.

\section{Lie-Poisson integration}

An important application of Corollary 3 is when the constraint surface is a Lie group which is also the symmetry group. In this case it shows that the constraint algorithm can provide a symplectic integrator for Lie-Poisson systems:

Corollary 4. Suppose that the Lie group $G$ is embedded as a submanifold of a linear space $M$, that $G$ acts linearly on $M$, and that $G$ is the level set of a left(right-) invariant constraint function $\phi: M \rightarrow V$. Suppose that the $G$-invariant extension to $T^{*} G$ of the Hamiltonian $H$ on $\mathfrak{g}^{*}$ satisfies the conditions of Corollary 3. If the constraint algorithm on $T^{*} M$ has a fixed point, then it produces a first order symplectic integrator for the Lie-Poisson system determined by $H$ and the $-(+)$ Lie-Poisson structure on $\mathfrak{g}^{*}$.

Many important groups in mechanics are defined as matrix groups and the constraint functions can be written as left or right invariant. For example, the rigid body (discussed below) has $G=S O(n)$ and $\phi(q)=q^{t} q-1$. In such a case one would take $M$ to be the space of $n \times n$ matrices. (Note that this is not a group, but no group structure on $M$ is needed by the constraint algorithm). Moreover, for any group $G$, Ado's theorem [30] provides an isomorphism, near the identity, of $G$ with a matrix group. This is all that is needed for the construction of symplectic integrators for Lie-Poisson systems. We do not know whether an invariant nondegenerate constraint function always exists globally.

Fortunately, even when presented with an arbitrary Lie algebra $\mathfrak{g}$, an isomorphism with a matrix Lie algebra is not required by Corollary 4 . What is needed is a realization of $G$ as a submanifold of a linear space $M$, as illustrated by the following example.

Example 1 A moment algebra. Many applications in physics involve the evolution of densities on a phase space $X$. For example, for the Vlasov-Poisson equation, the Lie algebra is the algebra of Hamiltonian functions on single particle phase space $X=\mathbb{R}^{3} \times \mathbb{R}^{3}$. The state variable is a density $\mu$ and can be represented by its moments $\left\langle\mu^{\alpha}\right\rangle \equiv \int_{X} x^{\alpha} \mu(x) d x$ where $\alpha$ is a multiindex. Truncating this infinite-dimensional Lie algebra is a difficult problem in general [27], but if the zeroth- and first-order moments are zero then then there is a straightforward approach. Let $\mathfrak{g}$ be the algebra of Hamiltonian functions with $\nabla h(0)=0$. Let $\mathfrak{i}_{n}$ denote the ideal in $\mathfrak{g}$ consisting of those functions whose derivatives up to order $n$ vanish at the origin. The quotient algebra $\mathfrak{g}_{n}=\mathfrak{g} / \mathfrak{i}_{n}$ is the image of the 
canonical projection $P_{n}$ which takes a function $f$ to the first $n$ terms in its Taylor series expansion. The bracket on $\mathfrak{g}_{n}$ is simply $[f, h]=P_{n}\{f, g\}_{X}$. A point in $\mathfrak{g}_{n}^{*}$ is the truncated moment description of a density $\mu$. Suppose that a Hamiltonian has been determined on $\mathfrak{g}_{n}^{*}$, say by the methods in Scovel and Weinstein [27]. To use the constraint algorithm we need to describe the Lie group $G_{n}$ of $\mathfrak{g}_{n}^{*}$ as a subset of a linear space $M$ defined by a $G_{n}$-invariant constraint function and for which the action of $G_{n}$ on $M$ is linear.

We take $M$ to be the linear space of polynomial maps

$$
M=\left\{A(x)=\sum_{|\alpha|=1}^{n-1} a_{\alpha} x^{\alpha}\right\}
$$

where $\alpha$ is a multiindex. It can be shown that $G_{n}$ is the subset of $M$ which satisfies the quadratic constraint

$$
\phi(A) \equiv P_{n-2}\left(d A J d A^{t}-J\right)=0
$$

- that is, $A \in G_{n}$ is an "almost symplectic" polynomial map. It is also true that the right action of $G_{n}$ on $M$ by composition of maps is linear (this is not true of the left action) and preserves the constraint function:

$$
(A B)(x)=P_{n-1} \sum_{\alpha} a_{\alpha}\left(\sum_{\beta} b_{\beta} x^{\beta}\right)^{\alpha}, \quad A \in M, B \in G_{n}
$$

is linear in the $a_{\alpha}$, and

$$
\phi(A B)=P_{n-2}\left(d A d B J d B^{t} d A^{t}-J\right)=P_{n-2}\left(d A J d A^{t}-J\right)=\phi(A)
$$

$\forall A \in M, B \in G_{n}$. We have not checked its nondegeneracy except for the case $n=2$, where it is nondegenerate. ${ }^{1}$

Theorem 2 and its corollaries determine equivariant symplectic integrators given that an invariant extension of the Hamiltonian exists. In many cases, such as the examples of section 6 , the extended Hamiltonian presents itself naturally as an unconstrained Hamiltonian. More generally, consider an Euler equation, a Lie-Poisson system with phase space the dual of a Lie algebra $\mathfrak{g}^{*}$ and a quadratic Hamiltonian determined from an inner product on $\mathfrak{g}$. Choose a complement of $\mathfrak{g}$ in $\mathfrak{g l}(n)$. The inner product can be extended to an inner product on $\mathfrak{g l}(n)$ for which $\mathfrak{g}$ and its complement are orthogonal. Let $\lambda_{q}^{*}: T_{q}^{*} G \rightarrow \mathfrak{g}^{*}$ be the pullback of left multiplication by $q$. Then the left-invariant extended Hamiltonian on $T^{*} G$ is

$$
H(q, p)=\frac{1}{2}\left\langle\lambda_{q}^{*} p, \lambda_{q}^{*} p\right\rangle
$$

where this inner product is the one induced on $\mathfrak{g l}(n)^{*}$ by that on $\mathfrak{g l}(n)$. Since this inner product is nondegenerate on the orthogonal complement to $\mathfrak{g}^{*} \subset \mathfrak{g l}(n)^{*}$, the Hamiltonian is cosymplectic with respect to $\phi$, and since the Hamiltonian is quadratic, the constraint algorithm has a fixed point. Of course, we could just as well have used right translations for right-invariant systems. We have therefore proved the following.

\footnotetext{
1 We note that since the constraints in this and many other cases are quadratic, any integrator that preserves quadratic invariants, such as some Runge-Kutta methods [4] and, in particular, the midpoint rule, can be used to approximate the exponential map and compute group elements.
} 
Theorem 5. Suppose that $G$ is determined by a nondegenerate left-invariant constraint function $\phi$. For an Euler equation on $\mathfrak{g}^{*}$, there exists an extended Euler Hamiltonian which is cosymplectic with respect to $\phi$. Therefore Corollary 4 can be used to construct a Lie-Poisson integrator. Furthermore the constraint algorithm (2,3) is a second-order method.

The second-order property will be shown in section 5 (see $(9,10)$ below).

We would like to point out that Theorem 5 solves an important open problem concerning Lie-Poisson integrators that we have discussed with $\mathrm{Li}$ [13] concerning the generation of the identity map on $\mathfrak{g}^{*}$. It is difficult to generate the identity from the Ge-Marsden generating function [6], because it is of the first kind. For some Lie algebras a Ge-Marsden generating function cannot generate the identity map at all [8]; for others a particular choice can only generate the identity in a neighborhood of the origin; and the identity map on $\mathfrak{g}^{*}$ is of the form $\operatorname{Ad}_{g(\mu)}^{*} \mu=\mu$ where $g(u) \in G$ is in the coadjoint isotropy group of $\mu$. One would prefer to have $g(\mu)=1$. The embedding in a Euclidean space considered in this paper allows the use of generating functions of the second kind, which easily generate the identity and have $g(\mu)=1$.

\section{The metric in holonomic constraints}

Often it is convenient to use a metric on $N$ which is the restriction of one on $M$ to map the dynamics to $T N$ and $T M$. For Hamiltonian systems with a cotangent lift symmetry group, the metric will have to be $G$-invariant in order that the new algorithms still preserve the momentum. In this way $\psi$ can be viewed as an orthogonal projection onto a subspace and the secondary constraints often take a simpler form.

Denote by $\sharp: T M \rightarrow T^{*} M$ the map induced from the metric and $b$ its inverse. Pull back the symplectic form and the Hamiltonian so that we can do Hamiltonian mechanics on the symplectic manifold $T M$. We use variables $(q, p)$ for the symplectic $T M$ and $(q, \dot{q})$ for the nonsymplectic $T M$. Define $d \phi^{t}=\left(d \phi^{*}\right)^{b}$ by the relation

$$
\langle\mu, d \phi \dot{q}\rangle=\left\langle d \phi^{t} \mu, \dot{q}\right\rangle
$$

which is zero when $\dot{q} \in T N$, because then $d \phi \dot{q}=0$. This gives the orthogonal decomposition

$$
T_{q} M=T_{q} N \oplus \operatorname{range}\left(d \phi^{t}\right),
$$

with $q \in N$. Applying the $\sharp$ operator gives the orthogonal decomposition

$$
T_{q}^{*} M=T_{q}^{*} N \oplus \operatorname{range}\left(d \phi^{*}\right)
$$

where $T N^{\sharp}$ has been identified as the natural representative of $T^{*} N$ in $T^{*} M$, since its orthogonal complement range $\left(d \phi^{*}\right)$ is annihilated by $\psi$. Therefore, $\psi$ is the orthogonal projection from the fibers of $\left.T^{*} M\right|_{N}$ to those of $T^{*} N$. The map $\psi$ transferred to $\left.T M\right|_{N}$ is the orthogonal projection onto $T N$ :

$$
(q, p) \mapsto\left(q,\left[1-d \phi^{t}\left(d \phi d \phi^{t}\right)^{-1} d \phi\right] p\right) .
$$

This satisfaction of the secondary constraints by a projection appears in [11] for the case of simple mechanical systems with the Euclidean metric. 
Consider now $C M$. The secondary constraints are

$$
\{\langle\phi, \mu\rangle, H\}=\left\langle d \phi\left(\partial_{p} H\right), \mu\right\rangle=\left\langle\partial_{p} H, d \phi^{t} \mu\right\rangle=0 \quad \forall \mu .
$$

Consequently, if the dynamical system is a simple mechanical system with $H=$ $\frac{1}{2}\left\langle p^{b}, p^{b}\right\rangle+V(q)$, then $\partial_{p} H=p^{b}$, so $C M$ is defined by

$$
\left\langle p^{b}, d \phi^{t} \mu\right\rangle=0
$$

so that $C M^{b}$ is identified with $T N$ and $C M$ with $T^{*} N$. In this case $\left.\psi\right|_{C M}$ is the identity map.

However, even for simple mechanical systems, often a metric is preferred which does not determine the kinetic energy. For example, in our treatment of the rigid body, we shall use the traditional metric $\langle X, Y\rangle=\frac{1}{2} \operatorname{tr}\left(X Y^{t}\right)$. More generally, since $M$ is a linear space, we may choose an inner product so that it may be identified with $\mathbb{R}^{n}$ with the Euclidean inner product. A computationally efficient choice would be to require $d \phi d \phi^{t}=1$, making the projection simpler. In these cases, $C M$ is not identified with $T^{*} N$ so the map $\left.\psi\right|_{C M}$ is nontrivial. We consider this more general case from now on.

To transform the algorithm $(2,3)$ to $T M$ one applies the isomorphism $b$ : $T^{*} M \rightarrow T M$. Denoting the gradient determined by the metric by $\nabla_{q} H=\partial_{q} H^{b}$, and the Hamiltonian induced from the original by the isomorphism $\sharp$ by $H$, in the algorithm one must replace $\partial$ by $\nabla$ and $d \phi^{*}$ by $d \phi^{t}$.

If one is given $(q, p) \in T N$, it might appear that the first step would be to reconstruct a $\hat{p} \in T_{q} M$ satisfying both the secondary constraints and $\psi(q, \hat{p})=p$. But because of the metric, the fibers of the symplectic $T N$ are the orthogonal complement to the range of $d \phi^{t}$. Therefore we can use the fact that for any $p$ in $T_{q} N$ and any $\hat{p}$ in $T_{q} M, \hat{p}-p=d \phi(q)^{t} \hat{\mu}$ for some $\mu$, so that we can absorb the difference in the second equation of the tangent formulation of (2). Likewise we can do the same for the $P$ variable in equation (3) giving rise to the following constraint algorithm on $T N$.

Begin with $(q, p) \in T N$. Solve

$$
\begin{aligned}
Q & =q+t \nabla_{p} H(q, \tilde{p}) \\
\tilde{p} & =p-t\left(\nabla_{q} H(q, \tilde{p})-d \phi(q)^{t} \mu\right) \\
\gamma(t): \quad \phi(Q) & =0 \\
P & =\psi(Q, \tilde{p})
\end{aligned}
$$

To conveniently construct higher-order methods from the map $\gamma(t)$, its adjoint map $\gamma^{-1}(-t)$ may be used. It is found by swapping $(q, p)$ with $(Q, P)$ and $t$ with $-t$, the result being

$$
\begin{aligned}
Q & =q+t \nabla_{p} H(Q, \tilde{p}) \\
\tilde{p} & =p+t d \phi(q)^{t} \mu \\
\gamma^{-1}(-t): \quad \phi(Q) & =0 \\
P & =\psi\left(Q, \tilde{p}-t \nabla_{q} H(Q, \tilde{p})\right)
\end{aligned}
$$

Note that if $H$ is a function of $p$ only, as in the free rigid body for example, then this is identical to the original map $\gamma(t)$. Thus in this case the constraint 
algorithm is symmetric, hence second order, automatically. In either case the order can be increased by composition of $\gamma\left(t_{i}\right)$ and $\gamma^{-1}\left(t_{i}\right)$ with appropriately chosen time steps $t_{i}[28,31,18]$.

If the Hamiltonian is separable, i.e., $H=T(p)+V(q)$, another second-order method is possible, namely RATTLE. It is usually formulated on $C M$ with $T(p)$ quadratic [11], but it can also be transferred to $T N$ :

$$
\begin{aligned}
p_{1} & =p-\frac{1}{2} t \nabla V(q) \\
Q & =q+t \nabla T\left(p_{2}\right) \\
p_{2} & =p_{1}+d \phi(q)^{t} \mu \\
\phi(Q) & =0 \\
p_{3} & =p_{2}-\frac{1}{2} t \nabla V(Q) \\
P & =\psi\left(Q, p_{3}\right)
\end{aligned}
$$

This map is symplectic since its equivalent version on $C M$ takes the form $(6,7)$ with the generating functions $S_{1}(q, \tilde{p})=\tilde{p}(q)+T(\tilde{p})+\frac{1}{2} V(q), S_{2}(Q, \tilde{p})=\frac{1}{2} V(Q)$. If $H$ is invariant under a linear cotangent lift symmetry, so are $T$ and $V$. Consequently, the proof that (11) is equivariant and momentum preserving is the same as in Theorem 2. A direct computation shows that the fixed point requirement can also be dropped.

Finally, for any of the algorithms there is an alternative way to formulate the step in which $\phi(Q)=0$ is enforced. When $H$ is separable, $\mu$ can be eliminated by applying $\psi$ : just solve

$$
\psi\left(q, \nabla T^{-1}(Q-q)\right)=t p_{1}, \quad \phi(Q)=0
$$

for $Q$, following which $p_{2}$ is given explicitly by

$$
p_{2}=\nabla T^{-1}((Q-q) / t) .
$$

This formulation is most useful when $T$ is quadratic as then (12) involves only linear transformations. This will be illustrated below on the rigid body.

\section{Examples}

In all the following examples, the primary constraints are quadratic and the secondary constraints can be extended to be quadratic also. Certain Runge-Kutta methods, such as the midpoint rule, preserve quadratic integrals [4] and are symplectic [24]. Consequently, one might guess that such methods could integrate these examples without a constraint algorithm by viewing the constraints as quadratic integrals of an appropriately extended Hamiltonian system. However, this does not appear to follow so easily. One needs an extension of the Hamiltonian from $C M$ to $T^{*} M$ which has the functions $\phi$ and $\phi_{2}$ as integrals. The obvious choice has dynamics which preserve all the level sets of $\phi$ but only the particular level set $\phi_{2}=0$ of $\phi_{2}$, not all its level sets. Indeed, for the rigid body, to leave all level sets invariant required a right invariance of the extended Hamiltonian which we were unable to obtain. 


\subsection{The free rigid body}

As an application of Corollary 4 we apply the constraint algorithm to the EulerArnol'd $n$-dimensional rigid body [20]. Here $M$ is the space of $n$ by $n$ matrices and $N$ is the special orthogonals $S O(n)$. The constraint function to the orthogonals $O(n)$ is

$$
\phi(q)=q^{t} q-1
$$

where 1 is the identity matrix. Since we are only interested in a neighborhood of $S O(n)$ we may use this function as a constraint function. We use the inner product $\langle X, Y\rangle=\frac{1}{2} \operatorname{tr}\left(X Y^{t}\right)$ as a metric on $T M$, on $T S O(n)$, and on $V=$ the symmetric matrices. (This is the conventional metric in rigid body studies, because under the standard isomorphism $\mathbb{R}^{3} \cong T_{q} S O(3)$ it coincides with the Euclidean metric on $\mathbb{R}^{3}$; however, it leads to some unusual factors of 2 on other spaces). With this inner product,

$$
T N=\left\{(q, p): q \in S O(n), q^{t} p \in \mathfrak{s o}(n)\right\} .
$$

The Lagrangian for the body subject to linear motions is

$$
L(q, \dot{q})=\frac{1}{2} \operatorname{tr}\left(\dot{q} \mathrm{~J} \dot{q}^{t}\right),
$$

where $\operatorname{tr}$ denotes the trace operator, and $\mathrm{J}$ may be taken to be diagonal with $\mathrm{J}_{i i}=\alpha_{i}$. In the case $n=3$, the principal moments of inertia of an actual rigid body are $\alpha_{2}+\alpha_{3}, \alpha_{1}+\alpha_{3}$, and $\alpha_{1}+\alpha_{2}$.

The Hamiltonian on $T M$ is

$$
H(q, p)=\frac{1}{8} \operatorname{tr}\left(p \mathrm{~J}^{-1} p^{t}\right)
$$

where we use the metric to identify $T M$ with $T^{*} M$. We have

$$
C M^{b}=\left\{(q, p): q \in S O(n), q^{t} p \mathrm{~J}^{-1} \in \mathfrak{s o}(n)\right\}
$$

and

$$
d \phi(q)^{t} \mu=2 q \mu
$$

where $\mu$ is a symmetric matrix. For completeness, we produce these calculations.

To compute $p=\partial_{\dot{q}} L$, take the variation of $L$ where $\delta \dot{q}$ is the variation of $\dot{q}$.

$$
\delta L=\frac{1}{2} \operatorname{tr}\left(\delta \dot{q} \mathrm{~J} \dot{q}^{t}\right)+\frac{1}{2} \operatorname{tr}\left(\dot{q} \mathrm{~J}(\delta \dot{q})^{t}\right)=\langle\delta \dot{q}, \dot{q} \mathrm{~J}\rangle+\langle\dot{q} \mathrm{~J}, \delta \dot{q}\rangle=\langle\delta \dot{q}, 2 \dot{q} \mathrm{~J}\rangle
$$

so that solving $p=2 \dot{q}$ J for $\dot{q}$ and substituting into the Lagrangian gives the described Hamiltonian. Similarly, $d \phi(q) \dot{q}=q^{t} \dot{q}+\dot{q}^{t} q$ and $\nabla_{p} H(q, p)=\frac{1}{2} p \mathrm{~J}^{-1}$, giving $C M^{b}$. Finally,

$$
\begin{aligned}
\left\langle d \phi(q)^{t} \mu, \delta q\right\rangle=\langle\delta \phi, \mu\rangle & =\frac{1}{2} \operatorname{tr}\left((\delta q)^{t} q \mu\right)+\frac{1}{2} \operatorname{tr}\left(q^{t} \delta q \mu\right) \\
& =\frac{1}{2} \operatorname{tr}\left((\delta q)^{t} q \mu\right)+\frac{1}{2} \operatorname{tr}\left(q \mu^{t} \delta q\right) \\
& =\langle 2 q \mu, \delta q\rangle,
\end{aligned}
$$

giving the formula for $d \phi(q)^{t}$. 
Let us show the equivalence described in Theorem 1 explicitly in this case. See Ratiu [20] for notation and a complete treatment of the geometry of the rigid body. The constrained Lagrangian can be written

$$
\begin{aligned}
L(q, \dot{q})=\frac{1}{2} \operatorname{tr}\left(\dot{q} \mathrm{~J} \dot{q}^{t}\right) & =\frac{1}{2} \operatorname{tr}\left(q^{t} \dot{q} \mathrm{~J}\left(q^{t} \dot{q}\right)^{t}\right) \\
& =\frac{1}{4} \operatorname{tr}\left(\mathrm{I}\left(q^{t} \dot{q}\right)\left(q^{t} \dot{q}\right)^{t}\right)
\end{aligned}
$$

where $\mathbf{I} a=a \mathrm{~J}+\mathrm{J} a$, the inertial tensor, is an invertible map on $\mathfrak{s o}(n)$. This latter form for the Lagrangian is preferable since the range of the Legendre transform is already $T^{*} S O(n)$ inside of $T^{*} M$ with respect to the decomposition (8).

The map I is the Hadamard product

$$
(\mathbf{I} a)_{i j}=\left(\alpha_{i}+\alpha_{j}\right) a_{i j}
$$

and has the inverse

$$
\left(\mathrm{I}^{-1} a\right)_{i j}=\frac{1}{\alpha_{i}+\alpha_{j}} a_{i j} .
$$

The Legendre transform is

$$
p=\partial_{\dot{q}} L=q \mathbf{l}\left(q^{t} \dot{q}\right)
$$

so that the Hamiltonian is

$$
H(q, p)=\frac{1}{4} \operatorname{tr}\left(\mathrm{I}^{-1}\left(q^{t} p\right)\left(q^{t} p\right)^{t}\right)
$$

and the equations of motion on $T N$ are

$$
\begin{aligned}
& \dot{q}=q \mathbf{l}^{-1}\left(q^{t} p\right) \\
& \dot{p}=p \mathbf{l}^{-1}\left(q^{t} p\right)
\end{aligned}
$$

where $q \in S O(n)$ and $p \in T_{q} S O(n)$, i.e., $q^{t} p \in \mathfrak{s o}(n)$.

One can obtain the Euler equations by letting $m=q^{t} p$ and computing

$$
\begin{aligned}
\dot{m} & =(\dot{q})^{t} p+q^{t} \dot{p} \\
& =-\mathrm{I}^{-1}(m) q^{t} p+q^{t} p \mathbf{I}^{-1}\left(q^{t} p\right) \\
& =\left[m, \mathrm{I}^{-1}(m)\right],
\end{aligned}
$$

where the square brackets denote the matrix commutator.

Now the Hamiltonian on $T M$ is

$$
H(q, p)=\frac{1}{8} \operatorname{tr}\left(p \mathrm{~J}^{-1} p^{t}\right)
$$

and so the unconstrained motion is given by

$$
\begin{aligned}
& \dot{q}=\frac{1}{2} p \mathrm{~J}^{-1} \\
& \dot{p}=0
\end{aligned}
$$


and the constrained motion is

$$
\begin{aligned}
& \dot{q}=\frac{1}{2} p \mathrm{~J}^{-1} \\
& \dot{p}=2 q \mu,
\end{aligned}
$$

where $\mu$ is a symmetric matrix determined by differentiating the constraint $q^{t} p \mathrm{~J}^{-1} \in \mathfrak{s o}(n)$.

These equations can now be pushed from $C M^{b}$ to $T N$. In this case

$$
\psi(q, p)=\left(q, \frac{1}{2}\left(p-q p^{t} q\right)\right) .
$$

Let $\operatorname{skew}(x)=\frac{1}{2}\left(x-x^{t}\right)$ so that $q^{t} p_{-}=\operatorname{skew}\left(q^{t} p\right)$. The equations of motion on $T N$ are

$$
\begin{aligned}
\dot{q} & =\frac{1}{2} p \mathrm{~J}^{-1} \\
\dot{p}_{-} & =\frac{1}{2}\left(\dot{p}-q \dot{p}^{t} q-\dot{q} p^{t} q-q p^{t} \dot{q}\right) \\
& =-\frac{1}{4}\left(p \mathrm{~J}^{-1} p^{t} q+q p^{t} p \mathrm{~J}^{-1}\right) \\
& =\frac{1}{4}\left(p q^{t} p \mathrm{~J}^{-1}-q p^{t} p \mathrm{~J}^{-1}\right) \\
& =\frac{1}{2} p_{-} q^{t} p \mathrm{~J}^{-1} .
\end{aligned}
$$

However, if $x \mathrm{~J}^{-1}$ is skew symmetric, then $\mathrm{I}\left(x \mathrm{~J}^{-1}\right)=x-x^{t}=2 \operatorname{skew}(x)$ so that $\mathrm{I}^{-1}(\operatorname{skew}(x))=\frac{1}{2} x \mathrm{~J}^{-1}$. Consequently, these equations become

$$
\begin{aligned}
\dot{q} & =\frac{1}{2} p \mathrm{~J}^{-1}=\frac{1}{2} q q^{t} p \mathrm{~J}^{-1}=q \mathrm{I}^{-1}\left(\operatorname{skew}\left(q^{t} p\right)\right)=q \mathrm{I}^{-1}\left(q^{t} p_{-}\right) \\
\dot{p}_{-} & =\frac{1}{2} p_{-} q^{t} p \mathrm{~J}^{-1}=p_{-} \mathrm{I}^{-1}\left(q^{t} p_{-}\right),
\end{aligned}
$$

which are the same as Eqs. (13). The demonstration of the equivalence is finished.

The constrained integrator is given on $C M$ in $(2,3)$, but we use the more simpler form on $T N(9)$. Let $(q, p) \mapsto(Q, P)$ be the integrator on $T N=T S O(n)$. First compute a symmetric matrix $\mu$ such that

$$
\begin{aligned}
Q & =q+\frac{t}{2} p_{2} \mathrm{~J}^{-1} \\
p_{2} & =p+2 t q \mu
\end{aligned}
$$

determines a $Q$ which satisfies $Q^{t} Q=1$. Then $P=\psi\left(Q, p_{2}\right)$, or

$$
P=\frac{1}{2}\left(p_{2}-Q p_{2}^{t} Q\right)
$$

We know from Corollary 4 that the map $(q, p) \mapsto(Q, P)$ reduces to a symplectic map on $\mathfrak{s o}(n)$. This symplectic integrator is

$$
m=q^{t} p \mapsto M=Q^{t} P .
$$


Theorem 6. This integrator is identical to the Moser-Veselov [19] integrator and therefore is integrable.

Proof. By Theorem 5 the momentum is preserved. Namely,

$$
P Q^{t}=p q^{t}
$$

which can be verified directly. Then,

$$
M=Q^{t} P=Q^{t} p q^{t} Q=\omega m \omega^{t}
$$

where $\omega=Q^{t} q$. This is the first component of their system.

The second component is determined by calculating

$$
\begin{aligned}
\omega^{t} \mathrm{~J}-\mathrm{J} \omega & =\mathrm{J}-2 t^{2} \mu+\frac{t}{2} q^{t} p_{1}-\mathrm{J}+2 t^{2} \mu-\frac{t}{2} p_{1}^{t} q \\
& =t \operatorname{skew}\left(q^{t} p_{1}\right)=t q^{t} p=t m
\end{aligned}
$$

The proof is finished.

This in fact corresponds to the second formulation of the implicit step, (12): $\nabla T^{-1}(Q-q)=2(Q-q) J$, and left-multiplying (12) by $q^{t}$ gives the MoserVeselov form. That this map is symmetric and hence second-order follows from the general considerations of Sec. 4 .

\subsection{The heavy top}

Including gravity in the above equations, to describe the heavy top, is straightforward. Let $c$ be the product of the acceleration due to gravity, the mass of the body, and the distance from the fixed point to the center of mass. The Hamiltonian on $T M$ is

$$
H(q, p)=\frac{1}{8} \operatorname{tr}\left(p \mathrm{~J}^{-1} p^{t}\right)+c \mathbf{k}^{t} q \boldsymbol{\chi}
$$

where $\mathbf{k}=(0,0,1)^{t}$ gives the direction of gravity and $\chi$ is a unit vector pointing from the fixed point to the center of mass at $t=0$. The derivation now follows as for the free rigid body; we are guaranteed that semi-direct reduction [16] applies to the integrator as it does to the continuous sytem. The reduced phase space is $\mathfrak{s e}(3) \cong \mathfrak{s o}(3) \times \mathbb{R}^{3} \ni(m, \mathbf{v})$ where $\mathbf{v}=q^{t} \mathbf{k}$. One obtains $(m, \mathbf{v}) \mapsto(M, \mathbf{V})$ where

$$
\begin{aligned}
\omega^{t} \mathrm{~J}-\mathrm{J} \omega & =t m-t^{2} c\left(\mathbf{v} \chi^{t}-\mathbf{v}^{t} \chi\right), \quad \omega \in S O(3) \\
\mathbf{V} & =\omega \mathbf{v} \\
M & =\left(\mathrm{J} \omega^{t}-\omega \mathrm{J}\right) / t
\end{aligned}
$$

and as before the attitude could be updated using $Q=q \omega^{t}$. (The last equation, following from (3), is also valid for the free rigid body, but obscures the fact that 
$m$ is being updated by a coadjoint action in that case.) We can also reduce the $T N$ version of RATTLE (11), obtaining

$$
\begin{aligned}
m_{2} & =m-\frac{1}{2} t c\left(\mathbf{v} \boldsymbol{\chi}^{t}-\mathbf{v}^{t} \boldsymbol{\chi}\right) \\
\omega^{t} \mathrm{~J}-\mathrm{J} \omega & =t m_{2}, \quad \omega \in S O(3) \\
\mathbf{V} & =\omega \mathbf{v} \\
m_{3} & =\left(\mathbf{J} \omega^{t}-\omega \mathrm{J}\right) / t \\
M & =m_{3}-\frac{1}{2} t c\left(\mathbf{V} \boldsymbol{\chi}^{t}-\mathbf{V}^{t} \boldsymbol{\chi}\right)
\end{aligned}
$$

The central feature to be dealt with in implementing integrators such as these is the need to solve equations in a group, here $S O(n)$. Parameterizing the group by the exponential map from its Lie algebra is very expensive, as functions such as $\exp \left(a d_{\xi}\right)$ need to be evaluated by Taylor series, multiplying the work per time step by a factor of about ten. Instead, we solve the constraint equations $\phi(q)=0$ iteratively; this is feasible if one can develop an iteration with convergence factor $\mathcal{O}(t)$. The formulation on $\mathfrak{s o}(n)$ is most convenient here, because the unknown $\omega$ is close to the identity. We write

$$
\omega=1+\omega^{a}+\omega^{s}, \quad \omega^{a} \text { antisymmetric, } \omega^{s} \text { symmetric }
$$

and note that $\omega^{a}$ is $\mathcal{O}(t)$ and $\omega^{s}$ is $\mathcal{O}\left(t^{2}\right)$. Then the following iteration has convergence factor $\mathcal{O}(t)$ :

$$
\begin{gathered}
\omega^{t} \mathrm{~J}-\mathrm{J} \omega=t m: \quad \omega_{i j}^{a} \leftarrow\left(\omega_{i j}^{s}\left(\alpha_{j}-\alpha_{i}\right)-t m_{i j}\right) /\left(\alpha_{i}+\alpha_{j}\right) \\
\omega^{t} \omega=1: \quad \omega^{s} \leftarrow-\frac{1}{2}\left(\omega^{s}+\omega^{a}\right)^{t}\left(\omega^{s}+\omega^{a}\right)
\end{gathered}
$$

Figure 1 illustrates a typical phase portrait of the heavy top as calculated by the second-order method. For the heavy top, the algorithm is not exactly energy-conserving as it was for the free rigid body. We take $\chi=(0,0,1), c=1$, $\alpha_{1}=0.44, \alpha_{2}=0.5, \alpha_{3}=1$ (parameters close to those of the integrable Lagrange top, discussed below), Casimirs $|\mathbf{v}|=1,-m_{23} v_{1}+m_{13} v_{2}-m_{12} v_{3}=0$, and define a Poincaré section on the energy surface $H=1$ by $v_{3}=0, \dot{v}_{3}>0$. This section is the ellipsoid $\frac{1}{4} \operatorname{tr}\left(\mathrm{I}^{-1}(m) m^{t}\right)=1$, the $m_{23}<0$ half of which is shown in Figure 1 . The existence of invariant circles in this section, even at the relatively large timestep $t=0.05$ (we took 60000 time steps for each initial condition), illustrates the conservation of the symplectic leaves and the symplecticity of the constraint algorithm on them.

For the Lagrange top, which has $\alpha_{1}=\alpha_{2}$ and $\chi=(0,0, \pm 1)^{t}$, the integrator is equivariant for the new right $S_{1}$-action and so preserves the momentum despite the fact that the constraint function is not right invariant. The proof is as follows. Because the constraints are not right invariant, the generating function equations are not equivariant under the group action. Consider the map determined by (2) without constraints (the situation is the same for $(3)$. Let $z=(q, p)$ and denote this map formally as

$$
Z=F(z, \mu)
$$


Instead of being equivariant as before, $F$ transforms as

$$
F\left(z g, g^{-1} \mu g\right)=F(z, \mu) g \text {. }
$$

Since the cosymplectic condition is satisfied, we can use the implicit function theorem to determine $\mu$ so that $\phi(F(z, \mu(z)))=0$. However, since $\phi$ transforms as $\phi(z g)=g^{-1} \phi(z) g$ at zero, it follows that $\phi(F(z g, \mu(z g)))=0$. Also,

$$
\phi\left(F\left(z g, g^{-1} \mu(z) g\right)\right)=\phi(F(z, \mu(z)) g)=g^{-1} \phi(F(z, \mu(z))) g=0,
$$

so that by the uniqueness property of the implicit function theorem, $\mu(z g)=$ $g^{-1} \mu(z) g$. Consequently,

$$
F(z g, \mu(z g))=\phi(F(z, \mu(z))) g
$$

and $F$ is equivariant.

The momentum corresponding to this $S_{1}$-action is $m_{12}$. Exact conservation of $m_{12}$ was confirmed numerically.

\subsection{The double spherical pendulum}

A detailed treatment of this system, including a derivation of its equations of motion, symmetry reductions, relative equilibria and their stability and bifurcations, may be found in Marsden [14, p.61]. The system consists of two spherical pendulums, the second attached to the first and the first attached to a fixed point, in a gravitational field. Let the position vectors of the individual pendulums relative to their joints be denoted $\mathbf{q}_{1}$ and $\mathbf{q}_{2}$ with fixed lengths $l_{1}$ and $l_{2}$, and let the masses be $m_{1}$ and $m_{2}$. The configuration space is $N=S_{l_{1}}^{2} \times S_{l_{2}}^{2}$, the product of spheres of radii $l_{1}$ and $l_{2}$. We embed $N$ in $M=\mathbb{R}^{3} \times \mathbb{R}^{3}$, use the Euclidean metric, and define the extended Hamiltonian on $T M$ by

$H\left(\mathbf{q}_{1}, \mathbf{q}_{2}, \mathbf{p}_{1}, \mathbf{p}_{2}\right)=\frac{1}{2 m_{1}}\left|\mathbf{p}_{1}-\mathbf{p}_{2}\right|^{2}+\frac{1}{2 m_{2}}\left|\mathbf{p}_{2}\right|^{2}+m_{1} g \mathbf{q}_{1} \cdot \mathbf{k}+m_{2} g\left(\mathbf{q}_{1}+\mathbf{q}_{2}\right) \cdot \mathbf{k}$

where $\mathbf{k}=(0,0,1)$ is the direction of gravity. The primary constraints are

$$
\frac{1}{2}\left(\mathbf{q}_{i} \cdot \mathbf{q}_{i}-l_{i}^{2}\right)=0 \quad i=1,2 .
$$

Denote this two-vector of constraints as $\phi: M \rightarrow \mathbb{R}^{2}$. Then,

$$
d \phi(\mathbf{q})(\mathbf{u}, \mathbf{v})=\left(\left\langle\mathbf{q}_{1}, \mathbf{u}\right\rangle,\left\langle\mathbf{q}_{2}, \mathbf{v}\right\rangle\right)
$$

which has the adjoint

$$
d \phi(\mathbf{q})^{t}\left(\mu_{1}, \mu_{2}\right)=\left(\mu_{1} \mathbf{q}_{1}, \mu_{2} \mathbf{q}_{2}\right) .
$$

The cosymplectic condition is satisfied because

$$
d \phi(\mathbf{q}) \frac{\partial^{2} H}{\partial \mathbf{p}^{2}} d \phi(\mathbf{q})^{t}=\left(\begin{array}{cc}
l_{1}^{2} / m_{1} & -\mathbf{q}_{1} \cdot \mathbf{q}_{2} / m_{1} \\
-\mathbf{q}_{1} \cdot \mathbf{q}_{2} / m_{1} & l_{2}^{2}\left(1 / m_{1}+1 / m_{2}\right)
\end{array}\right)
$$

is nonsingular. 
The definition of $C M^{b}$ is

$$
\begin{gathered}
\mathbf{q}_{1} \cdot \mathbf{q}_{1}=l_{1}^{2} \\
\mathbf{q}_{2} \cdot \mathbf{q}_{2}=l_{2}^{2} \\
\left(\mathbf{p}_{1}-\mathbf{p}_{2}\right) \cdot \mathbf{q}_{1}=0 \\
\left(\frac{1}{m_{1}}\left(\mathbf{p}_{2}-\mathbf{p}_{1}\right)+\frac{1}{m_{2}} \mathbf{p}_{2}\right) \cdot \mathbf{q}_{2}=0
\end{gathered}
$$

and of $T N$ is

$$
\begin{aligned}
& \mathbf{q}_{i} \cdot \mathbf{q}_{i}=l_{i}^{2} \\
& \mathbf{p}_{i} \cdot \mathbf{q}_{i}=0
\end{aligned} \quad(i=1,2)
$$

so

$$
\psi\left(\mathbf{q}_{1}, \mathbf{q}_{2}, \mathbf{p}_{1}, \mathbf{p}_{2}\right)=\left(\mathbf{q}_{1}, \mathbf{q}_{2}, \mathbf{p}_{1}-\frac{\left\langle\mathbf{p}_{1}, \mathbf{q}_{1}\right\rangle}{l_{1}^{2}} \mathbf{q}_{1}, \mathbf{p}_{2}-\frac{\left\langle\mathbf{p}_{2}, \mathbf{q}_{2}\right\rangle}{l_{2}^{2}} \mathbf{q}_{2}\right) .
$$

(This is an instance in which $d \phi d \phi^{t}=1$, making $\psi$ particularly simple.) The algorithm on $T N$ now applies directly. The implicit step reads

$$
\begin{aligned}
\mathbf{Q} & =\mathbf{q}+t M \tilde{\mathbf{p}} \\
\tilde{\mathbf{p}}_{i} & =\mathbf{p}_{i}+\mu_{i} \mathbf{q}_{i}, \quad i=1,2 \\
\mathbf{Q}_{i} \cdot \mathbf{Q}_{i} & =l_{i}^{2}, \quad i=1,2
\end{aligned}
$$

where $M=\left(\begin{array}{cc}1 / m_{1} & -1 / m_{2} \\ -1 / m_{2} & 1 / m_{1}+1 / m_{2}\end{array}\right)$.

The double spherical pendulum has an $S^{1}$ symmetry given by rotation about the vertical axis. The extended Hamiltonian $H$ is invariant under this symmetry, so the algorithm is equivariant. The momentum corresponding to this symmetry is $[14$, p.63]

$$
\mathbf{k} \cdot\left(\mathbf{q}_{1} \times \mathbf{p}_{1}+\mathbf{q}_{2} \times \mathbf{p}_{2}\right)
$$

(both on $C M^{b}$ and on $T N$ ) and we checked numerically that the algorithm did conserve this quantity.

In general, one must invert at least the linear part of the implicit equations to get $\mathcal{O}(t)$ convergence rates. For the rigid body this was trivial because the linear part is a Hadamard product; for the double spherical pendulum we used the simplest Newton-secant iteration, one Newton step with the same L-U decomposition used on succeeding steps.

We also checked our comment about the midpoint rule and quadratic integrals (see the start of this section) by determining the Lagrange multipliers analytically and integrating the resulting canonical system with the midpoint rule. As expected, the quadratic functions $\phi(q)$ and $d \phi(q)\left(\partial_{p} H\right)$ were not integrals of the resulting system, and their zero level set, although invariant under the dynamics, was not conserved by the midpoint rule. Both methods are symplectic, second order, and have roughly comparable truncation errors; but drifting of constraints under the midpoint rule lead to a much greater pointwise error in its solution, as shown in Figure 2. 


\section{References}

1. Abraham, R., and Marsden, J., Foundations of Mechanics, 2nd edition, Benjamin/Cummings, Reading, 1978.

2. Andersen, H. C., Rattle: a 'velocity' version of the shake algorithm for molecular dynamics calculations, J. Comp. Phys. 52 (1983), 24-34.

3. Channell, P. J., and Scovel, J. C., Integrators for Lie-Poisson dynamical systems, Physica $D 50$ (1991), 80-88.

4. Cooper, G. J., Stability of Runge-Kutta methods for trajectory problems, IMA J. Num. Anal. 7 (1987), 1-13.

5. Feng, K., and Ge, Z., On the approximation of linear H-systems, J. Comput. Math. 6 (1988), 88-97.

6. Ge, Z., and Marsden, J., Lie-Poisson Hamilton-Jacobi theory and Lie-Poisson integrators, Phys. Lett. A 133 (1988), 135-139.

7. Ge, Z., Equivariant symplectic difference schemes and generating functions, Physica D 49 (1991), 376-386.

8. Ge, Z., Generating functions, Hamilton-Jacobi equations and symplectic groupoids on Poisson manifolds, Indiana Univ. Math. J. 39(3) (1990), 859-876.

9. Guillemin, V. and Sternberg, S., Symplectic Techniques in Physics, Cambridge University Press, Cambridge, 1984.

10. Jay, L., Symplectic partitioned Runge-Kutta methods for constrained Hamiltonian systems (1993), Univ. de Genève preprint.

11. Leimkuhler, B. J., and Skeel, R. D., Symplectic numerical integration in constrained Hamiltonian systems (1993), preprint.

12. Lewis, D., and Simo, J. C., Conserving algorithms for the dynamics of Hamiltonian systems on Lie groups (1993), preprint.

13. Li, S., personal communication.

14. Marsden, J., Lectures on Mechanics, London Mathematics Society Lecture Note Series 174, Cambridge University Press, Cambridge, 1992.

15. Marsden, J. and Ratiu, T., Reduction of Poisson manifolds, Lett. Math. Phys. 11 (1986), $161-169$.

16. Marsden, J., and Ratiu, T., An Introduction to Mechanics and Symmetry, preprint, 1992.

17. McLachlan, R. I., Explicit Lie-Poisson integration and the Euler equations, Phys. Rev. Lett. 71 (1993), 3043-3046.

18. McLachlan, R. I., On the numerical integration of ordinary differential equations by symmetric composition methods, SIAM J. Sci. Comp. (1994), to appear.

19. Moser, J., and Veselov, A. P., Discrete versions of some classical integrable systems and factorization of matrix polynomials, Comm. Math. Phys. 139 (1991), 217-243.

20. Ratiu, T., The motion of the free $n$-dimensional rigid body, Indiana Univ. Math. J. 29:4 (1980), 609-629.

21. Reich, S., Symplectic integration of constrained Hamiltonian systems by Runge-Kutta methods (1993), Tech. report 93-13, Univ. British Columbia.

22. Reich, S., Numerical integration of the generalized Euler equations, preprint, 1993.

23. Reich, S., Momentum preserving symplectic integrators (1993), preprint.

24. Sanz-Serna, J. M., Runge-Kutta schemes for Hamiltonian systems, BIT 28 (1988), 877883.

25. Sanz-Serna, J. M., Symplectic integrators for Hamiltonian problems: an overview, Acta Numerica 1 (1992), 243-286.

26. Scovel, J. C., Symplectic numerical integration of Hamiltonian systems, in The Geometry of Hamiltonian Systems, ed. Tudor Ratiu, MSRI Publ. 22, 463-496, Springer-Verlag, New York, 1991.

27. Scovel, C., and Weinstein, A., Finite dimensional Lie-Poisson approximations to VlasovPoisson equations (1993), to appear in Comm. Pure Appl. Math.

28. Suzuki, M., Fractal decomposition of exponential operators with applications to manybody theories and Monte-Carlo simulations, Phys. Lett. A 146 (1990), 319-323.

29. Symon, R. K., Mechanics, Addison-Wesley, Reading MA, 1971. 
30. Varadarajan, V.S., Lie Groups, Lie Algebras, and Their Representations, Springer-Verlag, New York, 1974.

31. Yoshida, H., Construction of higher order symplectic integrators, Phys. Letters A $\mathbf{1 5 0}$ (1990), 262-268.

32. Yoshida, H., Recent progress in the theory and application of symplectic integrators, Cel. Mech. Dyn. Astr. 56 (1993), 27-43.

Figure 1. Orthogonal projection of an ellipsoidal Poincaré section of the heavy top. Coordinates are $m_{13}$ and $m_{12}$, so that the phase portrait for the nearby Lagrange top consists of horizontal lines.

Figure 2. Error growth in the free double spherical pendulum, two symplectic methods compared. Top: error in $\mathbf{q}(t)$ using the midpoint rule applied to the reduced equations; dots: error in the primary constraints under the midpoint rule; bottom: error in $\mathbf{q}(t)$ using the second-order constraint algorithm. 